\title{
Daily caffeine use and the sleep of college students
}

\author{
ROBERT A. HICKS, GREGORY J. HICKS, JOSEPH R. REYES, \\ and YVONNE CHEERS \\ San Jose State University, San Jose, California 95192
}

\begin{abstract}
The relationships between daily caffeine consumption and both habitual sleep duration and sleep satisfaction were assessed using the responses of 170 students. Essentially, our data replicated an earlier study, in that we found an inverse relationship between level of daily consumption of caffeinated drinks and habitual sleep duration and a nonsignificant relationship between caffeine use and sleep satisfaction.
\end{abstract}

In the treatment of insomnia, it is typically suggested that the patient refrain from consuming caffeinated drinks (Hicks, in press; Kales, Soldatos, \& Kales, 1981). While this assertion makes sense and is probably correct, the real-life evidence for the validity of this frequently offered advice is surprisingly sparse.

Recently, both Conway, Vickers, Ward, and Rahe (1981) and Gilliland and Andress (1981) provided indirect evidence that suggests that caffeine use is associated with state variables that are commonly implicated in the development of insomnia. That is, Gilliland and Andress found that moderate and heavy caffeine users reported significantly higher levels of anxiety than nonusers, and Conway et al. reported that caffeine use increased directly as a function of perceived occupational stress.

In an older study, Hollingworth (1912) reported data that suggest a direct relationship between the amount of caffeine consumed and sleep duration. Specifically, subjects were asked to keep sleep diaries, which included daily ratings of sleep satisfaction, subsequent to the ingestion of various amounts of caffeine. When compared to the control night, the sleep duration of these subjects was inversely related to the amount of caffeine consumed and was shortened by approximately $1 \mathrm{~h}$ (from $7.52 \mathrm{~h}$ to $6.59 \mathrm{~h}$ ) under the high $(6-\mathrm{g})$ caffeine treatment condition. It is surprising to note that, overall, the various levels of caffeine used failed to reduce sleep satisfaction. However, after the ingestion of $6 \mathrm{~g}$ of caffeine, there was a clear tendency to report sleep as "less satisfying." Thus, these data are somewhat ambiguous with regard to their implications for the suspected relationship between caffeine use and insomnia. That is, while sleep was shortened in a dose-related manner by caffeine, dissatisfaction with sleep did not increase until a relatively large dose of caffeine was administered to these subjects.

Please address requests for reprints to Robert A. Hicks, Department of Psychology, San Jose State University, San Jose, California 95192. In part, this research was supported by NIH Grant RR 08192-03 and NIMH Grant MH 17057-01.
Unfortunately, there are problems with the design of Hollingworth's (1912) research that render any interpretation of these data tenuous. To explain, Hollingworth used only 10 subjects in a repeated-measures design. Half of these subjects were habitually either light users or nonusers of caffeine. Further, the data were not tested for significance, nor were they reported in sufficient detail to permit others to perform these analyses. Therefore, the reliability of Hollingworth's results is open to question, as is their application to real-life situations. Thus, this study was designed to assess the relationships among daily caffeine consumption, habitual sleep duration, and overall sleep satisfaction in a manner that would serve as a constructive replication of Hollingworth's seminal study.

\section{METHOD}

To test the hypothesis that there is a relationship among habitual sleep duration, overall sleep satisfaction, and daily caffeine consumption, we asked 170 university undergraduate students to respond to a health-habits questionnaire that contained these critical questions: "Estimate the number of hours of sleep that you usually get each night," "Are you generally satisfied with your sleep?", and "Indicate the frequency with which you consume each of the following beverages: Coffee cups/day; Tea cups/day; Cola drinks

glasses/day." The responses to the last question in this set were used to provide a rough index of an individual's daily consumption of caffeine. This was done by summing the number of units of caffeinated drinks consumed each day and then treating this value as a score.

\section{RESULTS AND DISCUSSION}

We examined the relationship between daily caffeine consumption and habitual sleep duration from each possible perspective. The mean number of units of daily caffeinated drinks as a function of habitual hours of sleep duration are listed in Table 1.

The data summarized in Table 1 were analyzed by computing a one-way analysis of variance $[\mathrm{F}(4,169)=$ 8.26, $\mathrm{p}<.001$; est. $\left.\omega^{2}=.144\right]$.

The mean hours of sleep per day as a function of 
Table 1

Mean Number of Caffeine Drinks per Day as a Function of Habitual Sleep Duration (HSD)

\begin{tabular}{ccccr}
\hline & \multicolumn{4}{c}{ Parameter } \\
\cline { 2 - 5 } (in Hours) & Mean & SD & Total N & ND \\
\hline$<6$ & 4.4 & 3.0 & 14 & 8 \\
6 & 3.6 & 2.7 & 43 & 26 \\
7 & 2.7 & 1.9 & 58 & 24 \\
8 & 1.7 & 1.5 & 45 & 12 \\
$>8$ & 1.2 & 1.2 & 10 & 0 \\
\hline
\end{tabular}

Note $-N D=$ number of students who reported dissatisfaction with their sleep.

Table 2

Mean Hours of Sleep per Night for Students Who Report Various Levels of Daily Caffeine Use

\begin{tabular}{ccrcr}
\hline & \multicolumn{4}{c}{ Parameter } \\
\cline { 2 - 5 } CD/Day & Mean & SD & Total N & ND \\
\hline $0-1$ & 7.5 & 1.0 & 60 & 19 \\
$2-3$ & 7.1 & .9 & 61 & 27 \\
$4-5$ & 6.8 & 1.0 & 27 & 13 \\
$6-7$ & 6.5 & .9 & 14 & 5 \\
$\geqslant 8$ & 5.8 & 1.0 & 8 & 6 \\
\hline
\end{tabular}

Note-CD/day $=$ number of caffeine drinks per day. $N D=$ number of students who reported dissatisfaction with their sleep.

daily consumption of caffeinated drinks are listed in Table 2 . These data were analyzed by computing a oneway analysis of variance $[\mathrm{F}(4,169)=8.92, \mathrm{p}<.001$; est. $\left.\omega^{2}=.157\right]$.

Collectively, these data clearly suggest that there is a correlation between daily caffeine use and hours of sleep. This relationship is quite pronounced at the extremes of the distributions shown in Tables 1 and 2 . That is, habitual short sleepers $(<6 \mathrm{~h})$ use 3.6 times more caffeine each day than the long sleepers $(>8 \mathrm{~h})$ in our sample. Further, heavy caffeine users ( $\geqslant 8$ units/ day) habitually get about $75 \%$ as much sleep as the group that uses little or no caffeine (0-1 unit/day).

To further elaborate the relationships between caffeine use and sleep, we also examined the relationships among hours of sleep, daily caffeine consumption, and sleep satisfaction. To do this, we formed separate satisfied-dissatisfied contingency tables for hours of sleep and number of caffeinated drinks. (The data needed to construct each of these contingency tables are given in Tables 1 and 2, respectively). The chi squares computed to test the differences between the satisfieddissatisfied distributions for each of these variables demonstrated that there is a significant relationship between sleep satisfaction and habitual sleep duration $\left[\chi^{2}(4)=18.85, p<.001\right]$ and a nonsignificant relationship between sleep satisfaction and daily caffeine use $\left[\chi^{2}(4)=6.99\right]$. The finding of a significant relationship between sleep satisfaction and habitual sleep duration was anticipated and simply confirms other studies (e.g., Hicks \& Pellegrini, 1982). The nonsignificant relationship that we computed between sleep satisfaction and level of daily caffeine use conforms to Hollingworth's (1912) data exactly. In addition to this nonsignificant overall relationship, we also noted a tendency for only the heavy caffeine users in our sample to be differentially dissatisfied with their sleep. Put differently, apparently only heavy caffeine users tend to perceive their level of insomnia as a sleep problem.

To conclude, we suspect that caffeine use may be part of what might be described as the "shortened-sleep syndrome." The aspect of this syndrome that is relevant here is the fact that chronic reductions in habitual sleep duration have the effect of increasing levels of energy and/or drive (Hicks \& Guista, 1982; Vogel, 1979). Therefore, the inverse relationship that we have demonstrated between habitual sleep duration and the use of a stimulant like caffeine is clearly consistent with certain aspects of this syndrome.

\section{REFERENCES}

Conway, T. L., Vickers, R. R., Ward, H. W., \& Rahe, R. H. Occupational stress and variation in cigarette, coffee, and alcohol consumption. Journal of Health and Social Behavior, 1981, 22, 155-165.

Gilliland, K., \& Andress, D. Ad lib caffeine consumption, symptoms of caffeinism, and academic performance. American Journal of Psychiatry, 1981, 138, 512-514.

Hicks, R. A. Helping Type A patients to sleep better. Medical Aspects of Human Sexuality, in press.

Hicks, R. A., \& Guista, M. The energy levels of habitual long and short sleepers. Bulletin of the Psychonomic Society, 1982, 19, 131-132.

Hicks, R. A., \& Pellegrini, R. J. Sleep problems and Type AB behavior in college students. Psychological Reports, 1982, 51, 196.

Hollingworth, H. L. The influence of caffein on mental and motor efficiency. Archives of Psychology, 1912, No. 22.

Kales, A., Soldatos, C. R., \& Kales, J. D. Sleep disorders: Evaluation and management in office setting. In S. Arieti (Ed.), American handbook of psychiatry (2nd ed., Vol. 7). New York: Basic Books, 1981.

Vogel, G. W. A motivational function of REM sleep. In R. Drucker-Colin, M. Shkurovich, \& M. B. Sterman (Eds.), The functions of sleep. New York: Academic Press, 1979.

(Received for publication December 21, 1982.) 